\title{
Circulating leptin and adiponectin and their relation to glucose metabolism in children with Crohn's disease and ulcerative colitis
}

\author{
Giorgos Chouliaras ${ }^{1,2}$, loanna Panayotou', Daphne Margoni', Emilia Mantzou', Panagiota Pervanidou', Yannis Manios ${ }^{3}$, \\ George P. Chrousos ${ }^{1,2}$ and Eleftheria Roma ${ }^{1}$
}

BACKGROUND: Crohn's disease (CD) and ulcerative colitis (UC) result in metabolic consequences. We assessed circulating leptin and adiponectin concentrations and examined their relations to glucose metabolism in children with CD and UC. METHODS: Circulating morning fasting concentrations of leptin, adiponectin, glucose, and insulin were measured in 32 children with CD and 18 children with UC. Insulin resistance (IR) and $\beta$-cell function were evaluated by the updated homeostatic model assessments (HOMA2-IR and HOMA2-B).

RESULTS: Leptin was positively related to $\mathrm{BMI} Z$-scores overall and in the $C D$ and the $U C$ subgroups $(P<0.001)$. A negative correlation between leptin and disease activity was observed in the entire population $(P=0.034)$ and in the UC $(P=0.03)$ group. None of the assessed parameters was related to adiponectin. Fourteen percent of the participants were insulin resistant (15.6\% in the CD group and $11.1 \%$ in the UC group), significantly more than expected $(P<0.001)$. Leptin was associated with HOMA2-IR (overall: $r=0.29, P=0.045$ ). Pathway analysis suggested that, overall, disease activity and BMI significantly affect leptin, which in turn is the only correlate of HOMA2-IR.

CONCLUSION: Disease activity was significantly and inversely related to leptin in children with inflammatory bowel disease (IBD). A significant proportion of the patients had increased IR, which is positively related to circulating leptin.

rohn's disease (CD) and ulcerative colitis (UC) are inflammatory conditions of the intestines, often collectively referred to as inflammatory bowel diseases (IBDs). They are characterized by a chronic course with significant morbidity and require long-term medications and care. It is now recognized that IBDs are not just diseases of the bowels, but rather systemic disorders with frequent extraintestinal manifestations (1). In children, such complications include, among others, altered body composition, malnutrition, and metabolic disturbances, with the last being probably the least studied. Endocrine dysfunction in pediatric IBD, although recognized for more than 30 y (2), has not been extensively investigated. Growth impairment, being the most frequent endocrine disorder encountered in pediatric IBD, has drawn research efforts mainly on the growth hormone-insulin-like growth factor-1 axis (3). Few studies, mainly in adults, have addressed the issue of glucose homeostasis in IBD, and the results have been, so far, indicative of increased insulin resistance (IR) in CD.

Leptin and adiponectin are pluripotent adipokines involved in several physiologic processes of the human body, mainly balancing energy expenditure and contributing to immunoregulation (4). With regard to leptin and glucose homeostasis, an unambiguous observation is derived from genetically leptin-deficient individuals, who demonstrate glucose intolerance and peripheral IR. Both disturbances are reversible after exogenous administration of leptin (5). However, this relation is reversed in cases of systemic inflammation, such as in metabolic syndrome, in which higher leptin concentrations are related to decreased tissue sensitivity to insulin. It is still unclear whether this association is due to resistance of peripheral tissues to leptin, or a true causal effect of leptin on insulin sensitivity in the context of systemic inflammation (6). Similarly, adiponectin and IR have been associated, with the latter being positively influenced by the former (7). A growing body of experimental and clinical data suggests that, in addition to its metabolic effects, leptin also plays a proinflammatory role. A recent hypothesis for IBD is that inflamed, "creeping," hypertrophic mesenteric fat, which is a feature of active, "aggressive," CD (8), secretes amounts of leptin and adiponectin that are disproportional to the total body fat and that this metabolic imbalance might contribute significantly to systemic inflammation (9). Cross-sectional studies have given contradicting findings on the variation of leptin and adiponectin in IBD patients and healthy controls, and their association with the pathophysiology of the disease remains unclear (10).

The main aim of this cross-sectional study was to evaluate parameters that are associated with leptin and adiponectin concentrations in children with CD and UC. In particular, high-sensitivity C-reactive protein (hs-CRP), a widely used index of inflammation in both clinical practice and basic research, and interleukin (IL)-6, one of the most important

${ }^{1}$ First Department of Pediatrics, University of Athens, Athens, Greece; ${ }^{2}$ Research Division of Endocrinology and Metabolic Diseases, Biomedical Research Foundation of the Academy of Athens, Athens, Greece; ${ }^{3}$ Department of Nutrition and Dietetics, Harokopio University, Athens, Greece. Correspondence: Giorgos Chouliaras (georgehouliaras@msn.com) 
acute-phase reactants, were used to determine the inflammatory status of our patients. Furthermore, we assessed IR and pancreatic $\beta$-cell function, and developed a hypothesis on the interplay among disease activity and other characteristics, the circulating adipokines leptin and adiponectin, and glucose homeostasis.

\section{RESULTS}

\section{Descriptive Statistics}

A total of 50 patients (32 CD, $18 \mathrm{UC}$ ) were included in the analysis. Age, gender, disease characteristics, and medications at the time of assessment are presented in Table 1. Table 2 shows BMI, IR, and $\beta$-cell function as assessed by the homeostatic model assessments (HOMA2-IR and HOMA2-B), leptin levels, and inflammation indexes in the study groups. No significant differences were detected for $\log$ (leptin), $\log$ (HOMA2-B), or $\log$ (HOMA2-IR), whereas BMI $z$-scores were higher in UC patients ( 0.22 vs. -0.45 in the $C D$ subgroup), although the result was not significant $(P=0.07)$. In relation to the markers of inflammation, CD patients showed significantly increased levels of high-sensitivity IL-6 (hs-IL6; $P=0.02$ ), and a similar trend was seen for hs-CRP, a result that was marginally nonsignificant $(P=0.06)$.

The ratio of insulin-resistant individuals (HOMA2-IR $>97.5$ th percentile) to the total number of patients was $7 / 50$ $(14 \%)$, significantly $(P<0.001)$ higher as compared with the expected, which would have been $2.5 \%$ if IR in the study population were comparable to the general population of Greek children. No statistically significant differences were found between CD and UC patients with respect to IR $(5 / 32,15.6 \%$ and $2 / 18,11.1 \%$, respectively; $P=0.9$ ). Only one individual (a female UC patient) had impaired $\beta$-cell function, which corresponds to $2.0 \%$ in the entire study sample and was not significantly different from the expected $(P=0.99)$. For the UC and

Table 1. Demographic data, disease characteristics, and medications at the time of the evaluation

\begin{tabular}{|c|c|c|c|c|}
\hline & Overall & $\mathrm{CD}(n=32)$ & $\mathrm{UC}(n=18)$ & $P$ value \\
\hline $\begin{array}{l}\text { Gender, females, } \\
n(\%)\end{array}$ & $31(6$ & $21(6$ & $10(5$ & $0.5^{\mathrm{a}}$ \\
\hline Age, years ${ }^{b}$ & $10.4 \pm 3.5$ & $10.7 \pm 3.3$ & $9.9 \pm 3.7$ & $0.6^{c}$ \\
\hline Disease activity ${ }^{\mathrm{b}}$ & $19.6 \pm 11.3$ & $16.4 \pm 7.8^{d}$ & $25.3 \pm 14.3^{e}$ & - \\
\hline $\begin{array}{l}\text { Disease status, in } \\
\text { remission, } n(\%)\end{array}$ & $14(28.0 \%)$ & $5(27.8 \%)$ & $9(28.3 \%)$ & $0.9^{\mathrm{a}}$ \\
\hline $\begin{array}{l}\text { Glucocorticoid } \\
\text { treatment }^{f}\end{array}$ & $18(36 \%)$ & $13(40.6 \%)$ & $5(27.8 \%)$ & $0.5^{\mathrm{a}}$ \\
\hline $\begin{array}{l}\text { Immunomodulators, } \\
n(\%)^{f, g}\end{array}$ & $17(34 \%)$ & 15 (46.9\%) & $2(11.1 \%)$ & $0.01^{\mathrm{a}}$ \\
\hline $\begin{array}{l}\text { Aminosalicylates, } \\
n(\%)^{f}\end{array}$ & 38 (76\%) & $26(81.3 \%)$ & $12(66.6 \%)$ & $0.8^{\mathrm{a}}$ \\
\hline $\begin{array}{l}\text { Enteral nutrition, } \\
n(\%)^{f}\end{array}$ & $13(26.0 \%)$ & $9(21.8 \%)$ & $4(22.2 \%)$ & $0.8^{\mathrm{a}}$ \\
\hline \multicolumn{5}{|c|}{$\begin{array}{l}\text { CD, Crohn's disease; PCDAI, Pediatric Crohn's Disease Activity Index; PUCAI, Pediatric } \\
\text { Ulcerative Colitis Activity Index; UC, ulcerative colitis. }\end{array}$} \\
\hline \multicolumn{5}{|c|}{  } \\
\hline
\end{tabular}

CD groups, the respective ratios were 5.5\% (95\% confidence interval: $0.1-27.2)$ and $0 \%$ (95\% confidence interval: $0.0-10.9)$, respectively $(P=0.36)$.

\section{Leptin Correlates in Children With IBD}

Individual correlations were computed to evaluate the associations between BMI, gender, disease activity, type of medication (enteral nutrition, steroids, immunomodulators), inflammation markers, and leptin levels. As expected, BMI $z$-scores were significantly and positively related to $\log$ (leptin) over the entire population: Pearson's $r=0.50, P<0.001$. However, in the stratified analysis this relation remained significant only for CD patients (CD: Pearson's $r=0.62, P<0.001$, UC: Spearman's $\rho=0.22, P=0.4$ ). This could be attributed to the relatively small sample size of the UC subgroup $(n=18)$. Of note, $\log ($ leptin) levels did not differ between genders (all $P$ values $>0.05$, data not shown). A negative trend between disease activity, as measured by the Pediatric Ulcerative Colitis Activity Index, and $\log$ (leptin) concentrations was found for UC patients (Spearman's $\rho=-0.51, P=0.03$ ) but not in the population as a whole or in the $\mathrm{CD}$ subgroup. The same results were obtained when we used a categorical characterization for our patients (in remission vs. active disease). Overall, as well as in the $\mathrm{CD}$ group, no association with $\log$ (leptin) was found ( $P$ value $>0.05)$. By contrast, UC patients in remission had significantly higher $\log$ (leptin) levels than those with active disease $(2.6 \pm 0.7$ vs. $0.9 \pm 1.5$, respectively; $P=$ 0.01). Neither type of medication (enteral nutrition, steroids, immunomodulators) nor index of systemic inflammation (erythrocyte sedimentation rate, hs-IL6, and hs-CRP) were

Table 2. BMI, HOMA2-IR, HOMA2-B, leptin, and inflammation indexes in the study population

\begin{tabular}{lcclc}
\hline & Overall & $\mathrm{CD}(n=32)$ & $\mathrm{UC}(n=18)$ & $P$ value $^{\mathrm{a}}$ \\
\hline BMI, kg/m & $18.4 \pm 4.1$ & $17.9 \pm 4.0$ & $19.2 \pm 4.3$ & - \\
BMI z-score & $-0.21 \pm 1.3$ & $-0.45 \pm 1.1$ & $0.22 \pm 1.5$ & 0.07 \\
HOMA2-IR & $1.26 \pm 0.91$ & $1.28 \pm 0.93$ & $1.23 \pm 0.90$ & - \\
Log(HOMA2-IR) & $0.05 \pm 0.6$ & $0.06 \pm 0.6$ & $0.03 \pm 0.6$ & 0.9 \\
HOMA2-B & $111.0 \pm 57.8$ & $118.7 \pm 62.1$ & $97.4 \pm 47.8$ & - \\
Log(HOMA2-B) & $4.6 \pm 0.5$ & $4.7 \pm 0.5$ & $4.4 \pm 0.6$ & 0.2 \\
Leptin, ng/ml & $7.3 \pm 9.6$ & $6.5 \pm 8.9$ & $8.8 \pm 10.8$ & - \\
Log(leptin, ng/ml) & $1.1 \pm 1.5$ & $0.9 \pm 1.5$ & $1.4 \pm 1.5$ & 0.4 \\
Adiponectin, & $13.2 \pm 7.1$ & $13.6 \pm 7.7$ & $12.5 \pm 6.2$ & - \\
ng/ml & & & & \\
Log(adiponectin, & $2.42 \pm 0.59$ & $2.45 \pm 0.60$ & $2.37 \pm 0.60$ & 0.7 \\
$\mu \mathrm{Mg} / \mathrm{ml})$ & & & & \\
hs-IL6, pg/ml & $3.8 \pm 3.2$ & $4.7 \pm 3.6$ & $2.2 \pm 1.6$ & 0.02 \\
hs-CRP, mg/l & $10.9 \pm 26.2$ & $15.8 \pm 32.0$ & $2.4 \pm 3.5$ & 0.06 \\
ESR, mm/1 h & $41.8 \pm 22.0$ & $45 \pm 22.5$ & $36.2 \pm 20.8$ & 0.2 \\
\hline
\end{tabular}

Data are presented as mean \pm SD.

$\mathrm{CD}$, Crohn's disease; ESR, erythrocyte sedimentation rate; $\mathrm{HOMA2-B}$, homeostatic model of assessment 2 - $\beta$-cell function; HOMA2-IR, homeostatic model of assessment 2 -insulin resistance; hs-CRP, high-sensitivity C-reactive protein; hs-IL6, high-sensitivity interleukin-6; UC, ulcerative colitis.

aMann-Whitney test. 
related to $\log ($ leptin), either in the overall or stratified analyses (all $P$ values $>0.05$, data not shown).

Taking into consideration the results of the univariate analysis, we ran three (overall, CD subgroup, UC subgroup) multiple linear regression models with $\log ($ leptin) as the dependent variable. The results are illustrated in Table 3 . In the overall and UC final models, disease activity and BMI $z$-scores were the only significant correlates of circulating $\log$ (leptin). In the CD subgroup, no such result was observed, and BMI $z$-score was the main and only significant factor associated with circulating $\log ($ leptin).

\section{Adiponectin Correlates in Children With IBD}

Adiponectin was not related to inflammation indexes (hs-CRP, hs-IL6, erythrocyte sedimentation rate), disease activity (Pediatric Ulcerative Colitis Activity Index and Pediatric Crohn's Disease Activity Index), type of medication (enteral nutrition, steroids, immunomodulators), BMI $z$-score, gender, or age, neither in the entire sample nor in the CD and UC subgroups (all $P$ values $>0.05$, data not shown).

Correlates of Glucose Homeostasis Indexes (HOMA2-IR, HOMA2-B) in Children With IBD

IR, as assessed by the $\log$ (HOMA2-IR) model, was significantly related to $\log$ (leptin) levels in the overall analysis (Pearson's $r=$ $0.29, P=0.045)$. After stratification, the association retained its positive direction; however, the statistical significance was lost (CD: Pearson's $r=0.33, P=0.068$, UC: Spearman's $\rho=0.31, P=$ 0.21 ). Adiponectin, gender, age, disease activity, BMI $z$-score, type of medication (enteral nutrition, steroids, immunomodulators), and inflammatory indexes did not significantly affect $\log$ (HOMA2-IR).

A positive, although marginally nonsignificant, association was found between $\beta$-cell function and $\log$ (leptin) in the overall analysis (Pearson's $r=0.26, P=0.068$ ). The same results were obtained in the CD (Pearson's $r=0.32, P=0.075$ ) but not in the UC subgroup (Spearman's $\rho=0.15, P=0.5$ ). hs-CRP was negatively correlated with $\log (\mathrm{HOMA} 2-\mathrm{B})$ (Pearson's $r=$ $-0.36, P=0.044)$ in CD patients but not in the UC and overall groups (both $P$ values $>0.05$ ). Adiponectin, age, gender,

Table 3. Final multiple linear regression models analyzing the effect of BMI and disease activity on log(leptin)

\begin{tabular}{lcc}
\hline Variable & $\begin{array}{c}\text { Regression } \\
\text { coefficient }(\beta)\end{array}$ & $P$ value \\
\hline UC subgroup model & -0.06 & \\
Disease activity (PUCAI) & 0.48 & 0.014 \\
BMI z-score & & 0.036 \\
CD subgroup model & 0.81 & \\
$\quad$ BMI z-score & & $<0.001$ \\
Overall model & -0.03 & \\
$\quad$ Disease activity (PUCAI, PCDAI) & 0.67 & $<0.034$ \\
$\quad$ BMI z-score & & \\
\hline CD, Crohn's disease; PCDAl, Pediatric Crohn's Disease Activity Index; PUCAI, Pediatric \\
Ulcerative Colitis Activity Index; UC, ulcerative colitis.
\end{tabular}

disease activity, type of medication (enteral nutrition, steroids, immunomodulators), and BMI $z$-score did not, significantly, influence $\log$ (HOMA2-B).

\section{Correlates of Leptin and HOMA2-IR or HOMA2-B as Assessed by Pathway Analysis}

To assess whether the findings of the relations between $\mathrm{BMI} /$ disease activity with $\log$ (leptin) and $\log$ (leptin) with $\log$ (HOMA2-IR) could be combined in one hierarchical model, we performed a pathway analysis. Again, three models (overall, CD, and UC) were implemented. The results are schematically presented in Figure $1 \mathrm{a}-\mathrm{c}$. In CD patients, BMI $z$-score was the only significant determinant of $\log$ (leptin), which in turn had a significant effect on $\log$ (HOMA2-IR). Results in the UC group showed the same significant effect of BMI $z$-score on $\log$ (leptin) and confirmed the independent impact of disease activity on $\log ($ leptin), which was shown in linear regression. Nevertheless, in UC patients log(leptin) was not associated with $\log$ (HOMA2-IR). Finally, in the overall model, which was fitted under the assumption of combining the activity indexes into one variable, several interesting outcomes were observed. As can be seen in Figure 1c, analyzing the combined data set revealed that disease activity holds an essential role through a direct effect on circulating log(leptin). a

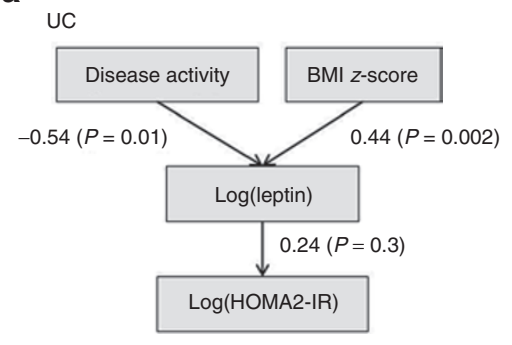

b $C D$

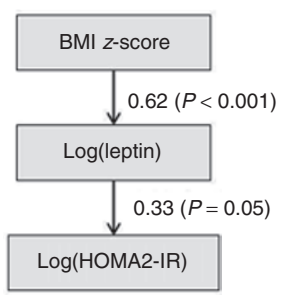

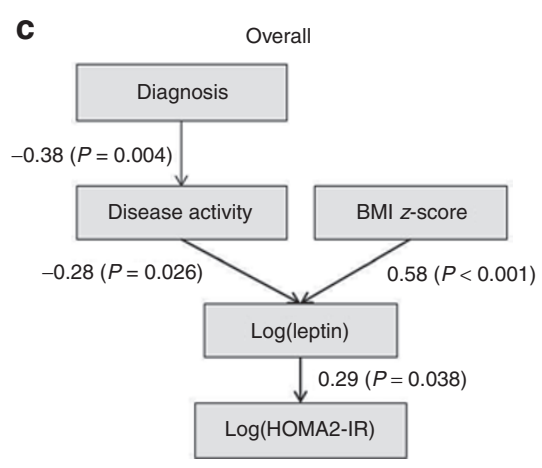

Figure 1. Schematic representation of the pathway analysis for HOMA2-IR, in (a) the UC subgroup, (b) the CD subgroup, and (c) the study sample as a whole. Numbers on arrows show the weighted regression coefficient for the respective relation as estimated by the models. Measures of fit for the three models are as follows: UC subgroup: RMSEA $<0.001, P_{\text {close }}=0.5, \chi^{2}=2.6$ (degrees of freedom $=3, P=0.5$ ); $C D$ subgroup: RMSEA $<0.001, P_{\text {close }}=0.4, \chi^{2}=0.75$ (degrees of freedom $=1, P=0.4$ ); overall: RMSEA $<0.001, P_{\text {close }}=0.8, \chi^{2}=2.52$ (degrees of freedom $=5, P=$ 0.8). CD, Crohn's disease; HOMA2-IR, homeostatic model of assessment 2-insulin resistance; RMSEA, root mean square error of approximation; UC, ulcerative colitis. 


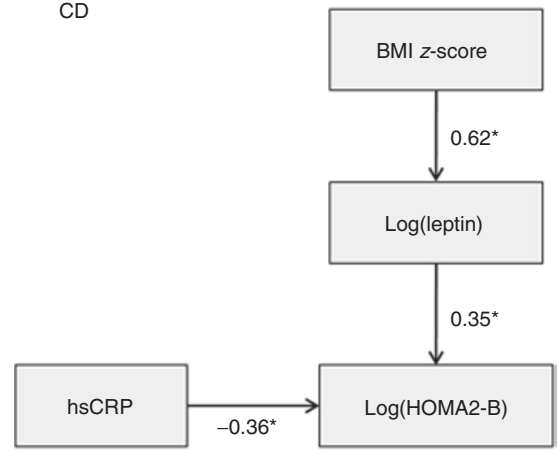

Figure 2. Schematic representation of the pathway analysis for HOMA2-B in $C D$ patients. Numbers on arrows show the weighted regression coefficient for the respective relation as estimated by the model. Measures of fit for the model: $C D$ subgroup: RMSEA $<0.001, P_{\text {close }}=0.7, \chi^{2}=1.47$ (degrees of freedom $=3, P=0.7$ ). ${ }^{*} P<0.05$. CD, Crohn's disease; HOMA2-B, homeostatic model of assessment $2-\beta$-cell function; hsCRP, high-sensitivity $\mathrm{C}$-reactive protein; RMSEA, root mean square error of approximation.

For HOMA2-B in $\mathrm{CD}$ patients, the pathway analysis showed a structure of relations similar to the one described for HOMA2-IR, with the addition of hs-CRP as a significant and independent factor related to HOMA2-B. Results are demonstrated in Figure 2. In the overall analysis, as well as in the UC subgroup, although the correlations of the univariate analysis were confirmed by the pathway analysis, none of the tested models fitted the data well and, therefore, in contrast to HOMA2-IR, no statistically robust hierarchical model could be proposed.

\section{DISCUSSION}

We assessed the metabolic profile (leptin, adiponectin, IR, and $\beta$-cell function) of children with IBD. Our results indicate that disease activity has a significant and negative effect on $\log$ (leptin) in UC, independent of BMI $z$-score. Of note, we did not find a similar effect in the CD subgroup, where only BMI was related to leptin. In our patients, no relation between leptin and gender, or leptin and age, was established, although such associations have been shown in normal subjects as well as in individuals with IBD $(11,12)$. The absence of such relations in this sample could be the result of the significant effect of disease activity.

Other reports have demonstrated conflicting results. In the first study of leptin in pediatric IBD, Hoppin et al. (13) reported similar levels of leptin among UC patients, CD patients, and controls, whereas the only significant correlates were BMI and gender. An interesting finding was an impressive, although not significant, increase of leptin levels in a subgroup of patients with active $\mathrm{CD}$ after achieving control of the disease. The leptin levels almost doubled, after the induction of remission, indicating that disease activity might play a significant role. In a prospective study (14) of children with $\mathrm{CD}$ on exclusive enteral nutrition, leptin levels were increased after $21 \mathrm{~d}$ of treatment, and this was observed with a parallel reduction of disease activity. In a recent pediatric study (12), children with CD had reduced circulating leptin levels as compared with controls, but no relations to disease activity or markers of inflammation were found. Karmiris et al. (15) showed that adult patients with UC had lower levels of leptin as compared with healthy controls, but no correlation with activity indexes was found. Several other groups showed opposite results, reporting no differences (16-18) or even increased (19) levels of leptin in IBD patients.

These inconsistent findings are difficult to explain if leptin is regarded solely as an appetite regulator. The pleiotropic nature of the molecule, which has both endocrine and immunoregulatory properties, might be the key to interpret those observations. The role of the mesenteric white adipose tissue in IBD has been proposed as the central player in the interplay between IBD and leptin (20). Experimental studies $(9,21)$ demonstrated increased leptin expression in mesenteric white adipose tissue specimens of IBD patients as compared with controls. This could result in inappropriately increased levels of leptin, leading to anorexia, malnutrition, and systemic inflammation. However, increased levels of leptin have not been consistently found in patients with IBD. In this frame, increased leptin is viewed as a "consequence" of IBD and probably the mediator between intestinal inflammation and malnutrition. Nevertheless, leptin possesses both pro- and anti-inflammatory properties, and the conflicting observations might indicate its role as an etiologic factor rather than an outcome of intestinal inflammation. The immunoregulatory aspects of leptin physiology have been demonstrated both at the systemic level as well as in the gastrointestinal tract. Leptin upregulates the secretion of inflammatory cytokines such as tumor necrosis factor- $\alpha$ (TNF- $\alpha$ ), IL-6, and IL-12 (22). Conversely, TNF- $\alpha$ and IL- $1 \beta$ enhance the expression of leptin mRNA in adipose tissue. This interaction forms a loop whose components influence each other in promoting inflammation (23). In addition, leptin negatively affects the proliferation of naturally occurring human $\mathrm{CD} 4^{+} \mathrm{CD} 25^{+} \mathrm{FoxP}^{+}$regulatory $\mathrm{T}$ cells, a class of great importance for immune tolerance in the gastrointestinal tract (24). At the cellular level, inflamed colonic epithelial cells in UC patients showed high expression of leptin, which is released in the lumen, resulting in injury of the epithelial cells and neutrophil infiltration (25). It is clear that no single, straightforward relation between leptin and IBD exists, and its importance in chronic intestinal inflammation remains unclear.

With regard to adiponectin, fewer studies-and those done only in adults-have assessed its significance in CD and UC. To our knowledge, no other data on adiponectin and pediatric IBD have been presented. In our population, adiponectin was not related to disease activity, anthropometric data, or gender. Three other groups have reached conflicting conclusions, in adult patients, reporting reduced adiponectin levels in IBD as compared with controls (18) and higher adiponectin concentrations in UC as compared with controls and CD $(15,26)$. Similarly to leptin, increased expression of adiponectin mRNA and increased release of adiponectin was found in hypertrophied mesenteric adipose tissue of CD patients $(21,27)$. The disagreement between the locally increased production of 
adiponectin in mesenteric fat and the variable peripheral measurements may reflect a topical, rather than systematic, mode of action and physiologic significance. Traditionally, adiponectin has been considered an anti-inflammatory adipokine (4). In particular, it has been shown to be able to suppress growth, maturation, and anti-TNFa and interferon- $\gamma$ production in macrophages treated with lipopolysaccharide (28). A feedback loop between adiponectin and proinflammatory mediators probably exists as well, given that TNF- $\alpha$ and IL- 6 are potent inhibitors of adiponectin gene expression (29). Overall, the experimental and clinical data are limited and at this point it is difficult to extract robust conclusions on the possible role of adiponectin in IBD.

Approximately $14 \%$ of children with IBD had peripheral IR as assessed by HOMA2-IR, a percentage that is more than five times higher than that of the general population. HOMA2-IR scores were positively and significantly related to leptin concentrations. By contrast, HOMA2-B in our sample did not deviate from the expected, indicating a normal $\beta$-cell function. In CD patients as well as in the overall analysis, leptin was the only significant correlate of HOMA2-IR, as predicted by pathway modeling. In children, no published data on the prevalence of IR exist. In adults, several studies have assessed the issue of glucose homeostasis. In 1999, Capristo et al. (30) found that whole-body glucose uptake and oxidation were similar between IBD patients and controls irrespective of disease activity. Subsequent studies, however, have consistently concluded that glucose homeostasis is affected in IBD, although their results are not directly comparable, due to the different methodology utilized in assessing insulin/glucose metabolism. Yorulmaz et al. (31) estimated that $27 \%$ of UC patients and $16.1 \%$ of CD patients had HOMA-IR $\geq 2.5$, i.e., IR. Despite the different method of HOMA-IR calculation and the arbitrary cut-off adopted in the latter study, the reported results agree with our findings of increased prevalence of IR in IBD. Three other groups $(18,32,33)$ also demonstrated hyperinsulinemia and reduced sensitivity to insulin in adults with $\mathrm{CD}$. The ability of anti-TNFa therapy to reverse glucose/insulin disturbances in CD has been supported by some studies but not by others.

The pathway analysis in our study provides a hypothetical sequence of events that might integrate our observations in a single pathophysiological model. The modeling suggests that BMI and disease activity simultaneously affect leptin, which, in turn, is the only factor associated with IR. The published data on the relation between leptin and insulin actions are conflicting. Although the leptin treatment-reversible IR of leptin-deficient mice and humans (34) is well established, experimental data support that leptin acts both as an insulin-sensitizing agent and as a contributor to the insulin-resistant phenotype (6). Our results indicate that in the context of a systemic inflammatory condition, such as IBD, the leptin-IR interaction may be altered because disease activity, perhaps through its downregulating effect on leptin, seems to increase sensitivity to insulin. This paradoxical observation has appeared before, given that two studies reached similar conclusions. Bregenzer et al. (32), mentioned that "patients with active disease showed significantly lower values for HOMA than patients with inactive disease," a conclusion identical to ours, whereas Valentini et al. (18) reversed the direction of the relation, suggesting that increased IR is protective of a relapse. These data taken together might indicate that inflammation is a modifier of the leptin-IR association.

In the CD subgroup, leptin and hs-CRP were positively and negatively correlated to $\beta$-cell function, respectively. Studies on healthy individuals have demonstrated a positive correlation between CRP levels and fasting glucose (35). To our knowledge, no other reports exist on the direct relation between hs-CRP and $\beta$-cell function. This finding requires further verification in future studies.

The main drawbacks of our study are the cross-sectional design of the analysis and the relatively small sample size, which might have prevented some comparisons from reaching statistical significance. Due to the cross-sectional nature of the study, the results of the pathway analysis should be considered as indications rather than absolute proofs of the underlying pathophysiology. The inclusion of a control would provide additional significant information; however, such data were not available.

In conclusion, disease activity may be a significant correlate of leptin concentrations in UC patients. A significant proportion of children with IBD have impaired glucose metabolism, and this study provides indications that this could be mediated by disease activity, perhaps through an effect on circulating leptin.

\section{METHODS}

This cross-sectional study was conducted in the First Department of Pediatrics of the University of Athens (Athens, Greece), following approval by the "Aghia Sophia" Children's Hospital Ethics Committee. Written informed consent was obtained by the parents of all participants and assent was obtained from the patients themselves.

Children with a diagnosis of CD or UC according to the Porto criteria (36) who were followed at the hospital outpatient clinic or hospitalized for a flare of the disease were eligible for inclusion. At the time of evaluation, disease characteristics and demographic and anthropometric data were collected. BMI was converted to BMI $z$-scores according to growth curves of the general Greek pediatric population. Disease activity was quantified using the Pediatric Crohn's Disease Activity Index (37) and Pediatric Ulcerative Colitis Activity Index (38). Both these indexes have been validated in pediatric populations with IBD, with higher values corresponding to higher disease activity. They combine clinical and laboratory data providing a single, continuous, arithmetic result ranging between 0 and 100 for the Pediatric Crohn's Disease Activity Index and between 0 and 85 for the Pediatric Ulcerative Colitis Activity Index. In both the scales, remission is defined as a score $<10$. Blood samples were drawn after overnight fasting, and the following parameters were measured in serum: glucose (mg/dl, Siemens Advia 1650 Clinical Chemistry System; Siemens Healthcare Diagnostics, Tarrytown, $\mathrm{NY}$ ), insulin (mIU/l, chemiluminescence, Siemens ACS180 System Analyzer; Siemens Healthcare Diagnostics), leptin (ng/ml, ELISA; Human Leptin ELISA BioVendor Research and Diagnostic Products, Heidelberg, Germany), adiponectin ( $\mu \mathrm{g} / \mathrm{ml}$, ELISA, AviBion Human Adiponectin (Acrp30); Orgenium Laboratories Business Unit, Vantaa, Finland), high-sensitivity C-reactive protein (mg/l, chemiluminescence (pg/ml), Immulite 2000; Siemens Heathcare Diagnostics), and high-sensitivity interleukin-6 (ELISA; Human IL-6 High Sensitivity Elisa Bender MedSystems, Vienna, Austria). Erythrocyte sedimentation rate $(\mathrm{mm} / 1 \mathrm{st}$ hour) was also measured from whole blood 
specimens. For the evaluation of IR and $\beta$-cell function, the updated HOMA2-IR and HOMA2-B, were utilized, respectively. HOMA2-IR and HOMA2-B values are computed using baseline (fasting) glucose and insulin measurements. Calculations were done by appropriate software as proposed by Wallace et al. and are considered more accurate than the linear solutions of the equations (39). The relation between HOMA2-IR and insulin sensitivity is reciprocal, with higher values of HOMA2-IR corresponding to decreasing sensitivity. A value of HOMA2-IR equal to 1 corresponds to $100 \%$ insulin sensitivity. Patients with baseline values outside the steady-state range (glucose: 3-25 mmol/l, insulin: 20-400 pmol/l) were excluded from the analysis. A patient was considered to have IR if his or her HOMA2-IR value was above the age- and gender-specific 97.5 th percentile of the general Greek population. Age- and gender-specific cut-offs were calculated using the HOMA2-IR and HOMA2-B models over a previously described data set of Greek children (40).

\section{Statistical Analysis}

All analyses were conducted both for the entire population and stratified according to the diagnosis. Continuous data are presented as mean \pm SD and compared by Student's $t$-test, or Mann-Whitney test, whenever the number of observations was $<30$. Correlations between continuous data were estimated by Pearson's correlation coefficient $(r)$, or Spearman's $\rho$. Categorical variables are presented as absolute $(n)$ and relative (\%) frequencies, and comparisons between groups were performed by Fisher's exact test. Leptin and adiponectin levels, HOMA2-B, and HOMA2-IR, as the main outcomes of interest, were logarithmically transformed to approximate normal distribution. Multiple linear regression analysis was used to assess correlates of circulating leptin, and results are presented as regression coefficients $(\beta)$.

Pathway analysis was used to simultaneously explore predictors of leptin levels and glucose homeostasis indexes (HOMA2-IR and HOMA2-B). Pathway analysis is a type of structural equation modeling. It is a multivariate statistical technique that examines hierarchical (causal) relations among two or more variables. The overall concept is based on testing a hypothetical pathway from exogenous variables (predictors) to endogenous variables (leptin, HOMA2-IR, and HOMA2-B in our analysis). In simple terms, pathway analysis permits simultaneous estimation of several different multiple regression equations. Standardized regression weights, along with $P$ values, are calculated for all specified relations in the diagram. The overall fit of the model to the observed data is assessed by $\chi^{2}$ test against the saturated (full) model. $P$ values $>0.05$ support a good fit. If the $P$ value of the $\chi^{2}$ test is $>0.05$, then the hypothesis that the proposed model fits the data equally well as the saturated model cannot be rejected. Another measure of goodness of fit is the root mean square error of approximation, which measures the discrepancy function obtained by fitting the model to the sample values. A root mean square error of approximation of about 0.05 or less indicates a close fit of the model. The probability that the computed root mean square error of approximation is not significantly over 0.05 is assessed by the measure $P_{\text {close' }}$ which should be $>0.05$.

A level of 0.05 or less was considered statistically significant. Analyses were performed using Stata 11.0 MP (Stata, College Station, TX) and AMOS 18.0 statistical software (SPSS, Chicago, IL).

\section{STATEMENT OF FINANCIAL SUPPORT}

No funding was received for this study.

Disclosure: The authors have no conflicts of interest to declare.

\section{REFERENCES}

1. Aloi M, Cucchiara S. Extradigestive manifestations of IBD in pediatrics. Eur Rev Med Pharmacol Sci 2009;13:Suppl 1:23-32.

2. Chong SK, Grossman A, Walker-Smith JA, Rees LH. Endocrine dysfunction in children with Crohn's disease. J Pediatr Gastroenterol Nutr 1984;3:529-34.

3. Vortia E, Kay M, Wyllie R. The role of growth hormone and insulinlike growth factor-1 in Crohn's disease: implications for therapeutic use of human growth hormone in pediatric patients. Curr Opin Pediatr 2011;23:545-51.

4. Stofkova A. Leptin and adiponectin: from energy and metabolic dysbalance to inflammation and autoimmunity. Endocr Regul 2009;43: $157-68$.

5. Kelesidis T, Kelesidis I, Chou S, Mantzoros CS. Narrative review: the role of leptin in human physiology: emerging clinical applications. Ann Intern Med 2010;152:93-100.

6. Ceddia RB, Koistinen HA, Zierath JR, Sweeney G. Analysis of paradoxical observations on the association between leptin and insulin resistance. FASEB J 2002;16:1163-76.

7. Díez JJ, Iglesias P. The role of the novel adipocyte-derived hormone adiponectin in human disease. Eur J Endocrinol 2003;148:293-300.

8. Erhayiem B, Dhingsa R, Hawkey CJ, Subramanian V. Ratio of visceral to subcutaneous fat area is a biomarker of complicated Crohn's disease. Clin Gastroenterol Hepatol 2011;9:684-687.e1.

9. Barbier M, Vidal H, Desreumaux P, et al. Overexpression of leptin mRNA in mesenteric adipose tissue in inflammatory bowel diseases. Gastroenterol Clin Biol 2003;27:987-91.

10. Karmiris K, Koutroubakis IE, Kouroumalis EA. Leptin, adiponectin, resistin, and ghrelin-implications for inflammatory bowel disease. Mol Nutr Food Res 2008;52:855-66.

11. Garcia-Mayor RV, Andrade MA, Rios M, Lage M, Dieguez C, Casanueva FF. Serum leptin levels in normal children: relationship to age, gender, body mass index, pituitary-gonadal hormones, and pubertal stage. J Clin Endocrinol Metab 1997;82:2849-55.

12. Aurangzeb B, Leach ST, Lemberg DA, Day AS. Assessment of nutritional status and serum leptin in children with inflammatory bowel disease. J Pediatr Gastroenterol Nutr 2011;52:536-41.

13. Hoppin AG, Kaplan LM, Zurakowski D, Leichtner AM, Bousvaros A. Serum leptin in children and young adults with inflammatory bowel disease. J Pediatr Gastroenterol Nutr 1998;26:500-5.

14. Bannerjee K, Camacho-Hübner C, Babinska K, et al. Anti-inflammatory and growth-stimulating effects precede nutritional restitution during enteral feeding in Crohn disease. J Pediatr Gastroenterol Nutr 2004;38:270-5.

15. Karmiris K, Koutroubakis IE, Xidakis C, Polychronaki M, Voudouri T, Kouroumalis EA. Circulating levels of leptin, adiponectin, resistin, and ghrelin in inflammatory bowel disease. Inflamm Bowel Dis 2006;12:100-5.

16. Nishi $\mathrm{Y}$, Isomoto $\mathrm{H}$, Ueno $\mathrm{H}$, et al. Plasma leptin and ghrelin concentrations in patients with Crohn's disease. World J Gastroenterol 2005;11:7314-7.

17. Ballinger A, Kelly P, Hallyburton E, Besser R, Farthing M. Plasma leptin in chronic inflammatory bowel disease and HIV: implications for the pathogenesis of anorexia and weight loss. Clin Sci 1998;94:479-83.

18. Valentini L, Wirth EK, Schweizer U, et al. Circulating adipokines and the protective effects of hyperinsulinemia in inflammatory bowel disease. Nutrition 2009;25:172-81.

19. Tuzun A, Uygun A, Yesilova Z, et al. Leptin levels in the acute stage of ulcerative colitis. J Gastroenterol Hepatol 2004;19:429-32.

20. Kaser A, Tilg H. "Metabolic aspects" in inflammatory bowel diseases. Curr Drug Deliv 2012;9:326-32.

21. Paul G, Schäffler A, Neumeier M, et al. Profiling adipocytokine secretion from creeping fat in Crohn's disease. Inflamm Bowel Dis 2006;12:471-7.

22. Faggioni R, Jones-Carson J, Reed DA, et al. Leptin-deficient (ob/ob) mice are protected from $\mathrm{T}$ cell-mediated hepatotoxicity: role of tumor necrosis factor alpha and IL-18. Proc Natl Acad Sci USA 2000;97:2367-72.

23. Faggioni R, Fantuzzi G, Fuller J, Dinarello CA, Feingold KR, Grunfeld C. IL-1 beta mediates leptin induction during inflammation. Am J Physiol 1998;274(1 Pt 2):R204-8.

24. De Rosa V, Procaccini C, Calì G, et al. A key role of leptin in the control of regulatory T cell proliferation. Immunity 2007;26:241-55.

25. Sitaraman S, Liu X, Charrier L, et al. Colonic leptin: source of a novel proinflammatory cytokine involved in IBD. FASEB J 2004;18:696-8.

26. Weigert J, Obermeier F, Neumeier M, et al. Circulating levels of chemerin and adiponectin are higher in ulcerative colitis and chemerin is elevated in Crohn's disease. Inflamm Bowel Dis 2010;16:630-7. 


\section{Articles | Chouliaras et al.}

27. Yamamoto K, Kiyohara T, Murayama Y, et al. Production of adiponectin, an anti-inflammatory protein, in mesenteric adipose tissue in Crohn's disease. Gut 2005;54:789-96.

28. Yokota T, Oritani K, Takahashi I, et al. Adiponectin, a new member of the family of soluble defense collagens, negatively regulates the growth of myelomonocytic progenitors and the functions of macrophages. Blood 2000;96:1723-32.

29. Ouchi N, Walsh K. Adiponectin as an anti-inflammatory factor. Clin Chim Acta 2007;380:24-30.

30. Capristo E, Mingrone G, Addolorato G, Greco AV, Gasbarrini G. Glucose metabolism and insulin sensitivity in inactive inflammatory bowel disease. Aliment Pharmacol Ther 1999;13:209-17.

31. Yorulmaz E, Adali G, Yorulmaz H, Ulasoglu C, Tasan G, Tuncer I. Metabolic syndrome frequency in inflammatory bowel diseases. Saudi J Gastroenterol 2011;17:376-82.

32. Bregenzer N, Hartmann A, Strauch U, Schölmerich J, Andus T, Bollheimer LC. Increased insulin resistance and beta cell activity in patients with Crohn's disease. Inflamm Bowel Dis 2006;12:53-6.

33. Bendet N, Scapa E, Cohen O, et al. Enhanced glucose-dependent glucagon-like peptide-1 and insulin secretion in Crohn patients with terminal ileum disease is unrelated to disease activity or ileal resection. Scand J Gastroenterol 2004;39:650-6.
34. Paz-Filho G, Wong ML, Licinio J. Ten years of leptin replacement therapy. Obes Rev 2011;12:e315-23.

35. Hashimoto K, Kasayama S, Yamamoto H, Kurebayashi S, Kawase I, Koga M. Strong association of C-reactive protein with body mass index and 2-h post-challenge glucose in non-diabetic, non-smoker subjects without hypertension. Diabet Med 2004;21:581-5.

36. IBD working Group of the European Society for Paediatric Gastroenterology, Hepatology and Nutrition. Inflammatory bowel disease in children and adolescents: recommendations for diagnosis--the Porto criteria. J Pediatr Gastroenterol Nutr 2005;41:1-7.

37. Hyams J, Markowitz J, Otley A, et al.; Pediatric Inflammatory Bowel Disease Collaborative Research Group. Evaluation of the pediatric crohn disease activity index: a prospective multicenter experience. J Pediatr Gastroenterol Nutr 2005;41:416-21.

38. Turner D, Hyams J, Markowitz J, et al.; Pediatric IBD Collaborative Research Group. Appraisal of the pediatric ulcerative colitis activity index (PUCAI). Inflamm Bowel Dis 2009;15:1218-23.

39. Wallace TM, Levy JC, Matthews DR. Use and abuse of HOMA modeling. Diabetes Care 2004;27:1487-95.

40. Manios Y, Moschonis G, Kourlaba G, et al. Prevalence and independent predictors of insulin resistance in children from Crete, Greece: the Children Study. Diabet Med 2008;25:65-72. 